\title{
Conformable Fractional Derivatives and It Is Applications for Solving Fractional Differential Equations
}

\author{
Ahmed Murshed Kareem \\ (University of Diyala, Iraq)
}

\begin{abstract}
This paper introduce two different definitions of fractional derivatives, namely Riemann-Liouville and Caputo derivatives, and some basics properties of these derivatives are discussed, conformable fractional derivativeare addressed.The paper focuses on the conditions needed in order to guarantee the general solution when we have the particular solution by applied some theorems.illustrated some examples.

Keywords:Gamma Function, Beta Function, Mittag-Leffler Function, Complementary Error Function, Fractional Integration, Riemann-Liouville derivative,fractional Caputo operator, conformable fractional derivative.
\end{abstract}

\section{Introduction}

Fractional differential equations are very important in many fields, like Biology, Physics, Mechanics, Electrochemistry of Corrosion, Engineering ,Viscoelasticity, Electrical Networks and Control Theory of Dynamic Systems[1,2]. Fractional calculus deals with integrals and derivatives of real or even complex order. It is a generalization of the classical calculus and therefore preserves some of the basic properties. As an intensively developing field if offers tremendous new features for research.The fractional calculus also finds applications in different fields of science, including theory of fractals, engineering, physics, numerical analysis, biology and economics. The history of fractional calculus started almost at the same time when classical calculus was established. It was first mention in Leibniz's letter to l' Hospital in 1695, where the idea of semi derivative was suggested. During time fractional calculus was built on formal foundations by many famous mathematicians e.g. Liouville, Riemann, Euler, Lagrange, Heaviside, Fourier, Abel etc[3,4,5]. The new definition reflects a nature extension of normal derivative which is called "conformable fractional derivative" $[6,7,8,9,10]$. The aim of my paper is to use conformable fractional derivative to solve fractional differential equation. The paper is organized as follows the special function as Gamma and Beta functions, Mittag-leffler function, , the complementary error function andFractional Integration are introduced in section 2.The two familiar operators of fractional calculus which are Rieman -Liouville and Caputo operators and the differences between these operators are introduced in section 3. The new definition of "conformable fractional derivatives" and some of its application are present in section 4 . The section 5 is devoted to the conclusion part.

\section{Special Functions}

Before we discuss the fractional Caputo and Riemann-Liouville derivatives, we present some special functions important for the fractional calculus as Gamma, Beta functions, the complementary error function and the Mittag-Leffler function. Furthermore, the fractional integration is introduced

\section{Gamma Function}

In the integer -order calculus the factorial plays an important role because it is one of the most fundamental combinatorial tools. The Gamma function has the same importance in the fractional - order calculus and it is basically given by the integral

$$
\Gamma(z)=\int_{0}^{\infty} t^{z-1} e^{-t} d t, \operatorname{Rez} .
$$

By analytic continuation, the function is extended to the total complex plane except for the points $\{0,-1,-2,-3,-$ $4,-5,-6, \ldots\}$ were it has simple poles. This $\Gamma: \mathbb{C} \backslash\{0,-1,-2,-3, \ldots\} \rightarrow \mathbb{C}$.

Below we show some basic properties of $\Gamma$ function, namely [1]:

$$
\begin{aligned}
& \Gamma(1)=\Gamma(2)=1, \\
& \Gamma(z+1)=z \Gamma(z)
\end{aligned}
$$

$\Gamma(\mathrm{z})=\frac{\Gamma(z+1)}{z}, \quad$ for negative value of $z$. $\Gamma(n)=(n-1) !, \quad n \in N_{0}$,

$\Gamma(n+1)=n !$, for $n \in N_{0}$,

Where $N_{0}$ is the set of the non-negative integers.From the above we can get: 
(a) $\Gamma\left(\frac{1}{2}\right)=\sqrt{\pi}$

(b) $\Gamma\left(\frac{5}{2}\right)=\frac{3}{2} \Gamma\left(\frac{3}{2}\right)=\frac{3}{2} \cdot \frac{1}{2} \Gamma\left(\frac{1}{2}\right)=\frac{3}{4} \sqrt{\pi}$

(c) $\Gamma\left(\frac{-3}{2}\right)=\frac{\Gamma\left(\frac{-3}{2}+1\right)}{\frac{-3}{2}}=\frac{\Gamma\left(\frac{-1}{2}\right)}{\frac{-3}{2}}=\frac{\Gamma\left(\frac{1}{2}\right)}{\frac{-3}{2} \cdot \frac{-1}{2}}=\frac{4}{3} \sqrt{\pi}$

\section{Beta Function}

The Beta function is very important for the computation of the fractional derivatives of the power function .It is defined by the two - parameter integral

$$
\beta(z, w)=\int_{0}^{1} t^{z-1}(1-t)^{w-1} d t, \text { Rez }>0, \text { Rew }>0(2)
$$

In addition $\beta(z, w)$ is used for convenience to replace a combination of Gamma functions. The relation between the Beta and Gamma function is [2]

$$
\beta(z, w)=\frac{\Gamma(z) \Gamma(w)}{\Gamma(z+W)}
$$

Equation (2.2) provides the analytical continuation of the Beta function to the entire complex plane via the analytical continuation of the Gamma function. It should also be mention that the Beta function is symmetric, namely:

\section{The Mittag -Leffler Function}

$$
\beta(z, w)=\beta(w, z) .
$$

While the Gamma function is a generalization of the factorial function, The Mittag-Leffler Function is a generalization of the exponential function, and it is one of the most important functions that are related to fractional differential equations. Firstly, we introduced a one-parameter function by using the series [2], namely $E_{\propto}(z)=\sum_{k=0}^{\infty} \frac{z^{k}}{\Gamma(\propto k+1)}, \quad \propto>0, \quad \propto \in R, z \in \mathbb{C}, \quad$ (4)

and then we define The Mittag-Leffler Function with two parameters, as:

$E_{\alpha, \beta}(z)=\sum_{k=0}^{\infty} \frac{z^{k}}{\Gamma(\alpha k+\beta)}, \quad \alpha, \beta>0, \propto, \beta \in R, z \in \mathbb{C},(5)$

Below we mentioned few of The Mittag-Leffler Function properties, namely:

$E_{1,1}(z)=\sum_{k=0}^{\infty} \frac{z^{k}}{\Gamma(k+1)}=\sum_{k=0}^{\infty} \frac{z^{k}}{k !},=e^{z}$,

$E_{1,2}(z)=\sum_{k=0}^{\infty} \frac{z^{k}}{\Gamma(k+2)}=\sum_{k=0}^{\infty} \frac{z^{k}}{(k+1) !}=\frac{1}{z} \sum_{k=0}^{\infty} \frac{z^{k+1}}{(k+1) !}=\frac{e^{z}-1}{z}$

$E_{1,3}(z)=\sum_{k=0}^{\infty} \frac{z^{k}}{\Gamma(k+3)}=\sum_{k=0}^{\infty} \frac{z^{k}}{(k+2) !}=\frac{1}{z^{2}} \sum_{k=0}^{\infty} \frac{z^{k+2}}{(k+2) !}=\frac{e^{z}-1-z}{z^{2}}$

In general,

$E_{1, m}(z)=\frac{1}{z^{m-1}}\left[e^{z}-\sum_{k=0}^{m-2} \frac{z^{k}}{k !}\right]$

Easily, we can obtain the following:

$E_{2,1}\left(z^{2}\right)=\sum_{k=0}^{\infty} \frac{z^{2 k}}{\Gamma(2 k+1)}=\sum_{k=0}^{\infty} \frac{z^{2 k}}{(2 k) !}=\cosh (\mathrm{z})$

$E_{2,2}\left(z^{2}\right)=\sum_{k=0}^{\infty} \frac{z^{2 k}}{\Gamma(2 k+2)}=\sum_{k=0}^{\infty} \frac{z^{2 k+1}}{z(2 k+1) !}=\frac{\sinh (z)}{z}$

$E_{2,1}\left(-z^{2}\right)=\sum_{k=0}^{\infty} \frac{\left(-z^{2}\right)^{k}}{\Gamma(2 k+1)}=\sum_{k=0}^{\infty} \frac{(-1)^{k} z^{2 k}}{(2 k) !}=\cos (\mathrm{z})$

$E_{2,2}\left(-z^{2}\right)=\sum_{k=0}^{\infty} \frac{\left(-z^{2}\right)^{k}}{\Gamma(2 k+2)}=\sum_{k=0}^{\infty} \frac{(-1)^{k} z^{2 k+1}}{z(2 k+1) !}=\frac{\sin (z)}{z}$ 
The Complementary Error Function

The complementary error function is an entire function defined as $\operatorname{erfc}(z)=\frac{2}{\sqrt{\pi}} \int_{z}^{\infty} e^{-t^{2}} d t$

Special values of the corresponding error function are given below

$\operatorname{erfc}(-\infty)=\frac{2}{\sqrt{\pi}} \int_{-\infty}^{\infty} e^{-t^{2}} d t=2$,

$\operatorname{erfc}(0)=\frac{2}{\sqrt{\pi}} \int_{-\infty}^{\infty} e^{-t^{2}} d t=1$,

$$
\operatorname{erfc}(+\infty)=\frac{2}{\sqrt{\pi}} \int_{-\infty}^{\infty} e^{-t^{2}} d t=0
$$

The following relations are interesting to be mentioned, namely

$\operatorname{erfc}(-x)=2-\operatorname{erfc}(x)$,

$\int_{0}^{\infty} \operatorname{erfc}(x) d x=\frac{1}{\sqrt{\pi}}$

$\int_{0}^{\infty} \operatorname{erfc}^{2}(x) d x=\frac{2-\sqrt{2}}{\sqrt{\pi}}$.

Respectively.

\section{Fractional integration}

In this subsection we recall the Cauchy's formula

$J^{n} f(t)=\int_{\mathrm{a}}^{\mathrm{t}} \int_{\mathrm{a}}^{\tau_{1}} \ldots \int_{\mathrm{a}}^{\tau_{\mathrm{n}-1}} f(\tau) \mathrm{d} \tau \ldots \mathrm{d} \tau_{2} \mathrm{~d} \tau_{1}=\frac{1}{(n-1) !} \int_{a}^{t} f(\tau)(t-\tau)^{n-1} d \tau$.

Definition1: Suppose that $\omega>0, t>a, \omega, a, t \in R$. Then, the fractional integration operatoris given by:

$J^{\omega} f(t)=\frac{1}{\Gamma(\omega)} \int_{a}^{t} f(\tau)(t-\tau)^{\omega-1} d \tau$.

We give some properties of the fractional integration by convention we have

$J^{0} f(t)=f(t), \quad(10)$

i.e. $J^{0}=1$ is the identity operator.

Another property is the linearity, namely:

$J^{\omega}(\Lambda f(t)+g(t))=\Lambda J^{\omega} f(t)+J^{\omega} g(t), \omega \in R_{+}, \Lambda \in \mathbb{C}$.

If $f(t)$ is continuous for all $t \geq 0$ the following equalities holds

$\lim _{\omega \rightarrow 0} J^{\omega} f(t)=f(t)$,

$J^{\omega}\left(J^{\beta} f(t)\right)=J^{\beta}\left(J^{\omega} f(t)\right)=J^{\omega+\beta} f(t), \quad \omega, \beta \in R_{+}, \Lambda \in \mathbb{C}$, respectively.

\section{Rieman-Liouville And Caputo Fractional Differential}

After the introduction of the fractional integration operator it is reasonable to define also the fractional differentiation operator. There are different definitions, which do not coincide in general.

Definition 2:Suppose that $\alpha>o, t>a, \alpha, a, t \in R$.Then we have

$D^{\alpha} f(t)=\left\{\begin{array}{c}\frac{1}{\Gamma(n-\alpha)} \frac{d^{n}}{d t^{n}} \int_{a}^{t} \frac{f(\tau)}{(t-\tau)^{\alpha+1-n}} d \tau, \quad n-1<\alpha<n \in N, \\ \frac{d^{n}}{d t^{n}} f(t), \quad \alpha=n \in N .\end{array}\right.$

This is named the Riemann-Liouville fractional derivative of order $\alpha$.

Theorem 1: The Riemann-Liouville derivative of order $\alpha>0$, with $n-1<\alpha<n$

Of the power function $f(t)=t^{p}$ for $\mathrm{p} \geq 0$ is given by

$D^{\alpha} t^{p}=\frac{\Gamma(p+1)}{\Gamma(p-\alpha+1)} t^{p-\alpha}$

Proof :We compute The Riemann-Liouville derivative of power $\alpha>o$ as :

$D^{\alpha} t^{p}=\frac{1}{\Gamma(n-\alpha)} \frac{d^{n}}{d t^{n}} \int_{0}^{t}(t-u)^{n-\alpha-1} u^{p} d u$

Set $u=v t$ for $0 \leq v \leq 1, d u=t d v$ and we get :

$D^{\alpha} t^{p}=\frac{1}{\Gamma(n-\alpha)} \frac{d^{n}}{d t^{n}} \int_{0}^{1}((1-v) t)^{n-\alpha-1}(v t)^{p} t d v$ 


$$
\begin{aligned}
& =\frac{1}{\Gamma(n-\alpha)} \int_{0}^{1}(1-v)^{n-\alpha-1} v^{p} d v \frac{d^{n}}{d t^{n}} t^{n+p-\alpha} \\
& =\frac{1}{\Gamma(n-\alpha)} \beta(p+1, n-\alpha) \frac{d^{n}}{d t^{n}} t^{n+p-\alpha} \\
& =\frac{1}{\Gamma(n-\alpha)} \beta(p+1, n-\alpha) \frac{\Gamma(n+p-\alpha+1)}{\Gamma(p-\alpha+1)} t^{p-\alpha} \\
& =\frac{1}{\Gamma(n-\alpha)} \frac{\Gamma(p+1) \Gamma(n-\alpha)}{\Gamma(n+p-\alpha+1)} \frac{\Gamma(n+p-\alpha+1)}{\Gamma(p-\alpha+1)} t^{p-\alpha} \\
& =\frac{\Gamma(p+1)}{\Gamma(p-\alpha+1)} t^{p-\alpha}
\end{aligned}
$$

Definition3:Suppose that $\alpha>0, t>a, \alpha, c, t \in R$. The fractional Caputo operator has the form

$$
D_{*}^{\alpha} f(t)=\left\{\begin{array}{l}
\frac{1}{\Gamma(n-\alpha)} \int_{a}^{t} \frac{f^{(n)}(\tau)}{(t-\tau)^{\alpha+1-n}} d \tau, n-1<\alpha<n \in N, \\
\frac{d^{n}}{d t^{n}} f(t), \\
\quad \alpha=n \in N .
\end{array}\right.
$$

Theorem 2 [6]:

Suppose $\mathrm{t}>0, \alpha \in R$, and $n-1<\alpha<n, n \in N$, then the following relation between the Riemann-Liouville and the Caputo operator holds:

$$
D_{*}^{\alpha} f(t)=D^{\alpha} f(t)-\sum_{k=0}^{n-1} \frac{t^{k-\alpha}}{\Gamma(k+1-\alpha)} f^{(k)}(0)
$$

Proof :The well-known Taylor series expansion about the point 0 reads as:

$$
\begin{aligned}
& f(t)=f(0)+t f^{\prime}(0)+\frac{t^{2}}{2 !} f^{\prime \prime}(0)+\frac{t^{3}}{3 !} f^{\prime \prime \prime}(0)+\cdots+\frac{t^{n-1}}{(n-1) !} f^{n-1}(0)+R_{n-1} \\
& =\sum_{k=0}^{n-1} \frac{t^{k}}{\Gamma(k+1)} f^{k}(0)+R_{n-1} .
\end{aligned}
$$

Considering (8) we conclude that

$R_{n-1}=\int_{0}^{t} \frac{f^{(n)}(\tau)(t-\tau)^{n-1}}{(n-1) !} d \tau=\frac{1}{\Gamma(n)} \int_{0}^{t} f^{(n)}(\tau)(t-\tau)^{n-1} d \tau=J^{n} f^{(n)}(t)$.

Now, by using the linearity characteristic of the Riemann-Liouville fractional derivative, we obtain:

$$
\begin{aligned}
& D^{\alpha} f(t)=D^{\alpha}\left(\sum_{k=0}^{n-1} \frac{t^{k}}{\Gamma(k+1)} f^{(k)}(0)+R_{n-1}\right) \\
& =\sum_{k=0}^{n-1} \frac{D^{\alpha} t^{k}}{\Gamma(k+1)} f^{(k)}(0)+D^{\alpha} R_{n-1} \\
& =\sum_{k=0}^{n-1} \frac{\Gamma(\mathrm{k}+1)}{\Gamma(k-\alpha+1)} \frac{t^{k-\alpha}}{\Gamma(k+1)} f^{(k)}(0)+D^{\alpha} J^{n} f^{n}(t) \\
& =\sum_{k=0}^{n-1} \frac{t^{k-\alpha}}{\Gamma(k-\alpha+1)} f^{k}(0)+J^{n-\alpha} f^{n}(t) \\
& =\sum_{k=0}^{n-1} \frac{t^{k-\alpha}}{\Gamma(k-\alpha+1)} f^{k}(0)+D_{*}^{\alpha} f(t) .
\end{aligned}
$$

This means that [6]:

$D_{*}^{\alpha} f(t)=D^{\alpha} f(t)-\sum_{k=0}^{n-1} \frac{t^{k-\alpha}}{\Gamma(k+1-\alpha)} f^{k}(0)$.

So, the proof is completed.

Remark: The difference between Caputo and Riemann - Liouville formulas for the fractional derivatives leads to the following differences.

a) Caputo fractional derivative of a constant equals zero while (Riemann - Liouville) fractional derivative of a constant does not equal zero. 
b) The non-commutation, in Caputo fractional derivative we have $D_{*}^{\alpha} D^{m} f(t)=D_{*}^{\alpha+m} f(t) \neq D^{m} D_{*}^{\alpha} f(t)(14)$

Where $\alpha \in(n-1, n), n \in N, m=1,2,3, \ldots$.

In general , the Riemann-Liouville derivative is also non-commutation as:

$D^{m} D^{\alpha} f(t)=D^{\alpha+m} f(t) \neq D^{\alpha} D^{m} f(t)$

Where $\alpha \in(n-1, n), n \in N, m=1,2,3, \ldots$

Note that the formulas as in (14) and (15) become equalities under the following additional conditions:

$f^{(s)}(0)=0, s=n, n+1, \ldots, m$. For $D_{*}^{\alpha}$ and

$f^{(s)}(0)=0, s=0,1,2, \ldots, m$, for $D^{\alpha}$

\section{Conformable Fractional Definition}

Definition4[4]:Given function $f:[0, \infty) \rightarrow R$. Then the (Conformable fractional derivative) of $f$ of order $\alpha$ is defined by :

$T_{\propto}(f)(t)=\lim _{\varepsilon \rightarrow 0} \frac{f\left(t+\varepsilon t^{1-\alpha}\right)-f(t)}{\varepsilon}$

For all $\mathrm{t}>0, \alpha \in(0,1)$, if $\stackrel{\varepsilon}{f}$ is $\propto$-differentiable in some $(0, \alpha) . \alpha>0$ and

$\lim _{t \rightarrow 0} f^{\propto}(t)$ exists, then define $f^{\propto}(0)=\lim _{t \rightarrow 0} f^{\propto}(t)$

We sometimes, write $f^{\propto}(t)$ for $T_{\propto}(f)(t)$, to denote the conformable fractional derivatives of $f$ of order $\alpha$. In addition, if the conformable fractional derivative of $f$ of order $\alpha$ exists, then we say $f$ is $\propto$-differentiable. We should take into consideration that $T_{\propto}\left(t^{p}\right)=p t^{p-\propto}$.Further, this definition coincides happen with the same of traditional definition of Riemann- Liouville and of Caputo on polynomials (up to a constant multiple).

Theorem 3 : (Conformable fractional derivative of known functions)

(1) $T_{\propto}\left(e^{c t}\right)=c t^{1-\propto} e^{c t}$

(2) $T_{\propto}(\sin (a t))=a t^{1-\propto} \cos (a t), a \in R$

(3) $T_{\propto}(\cos (a t))=-a t^{1-\alpha} \sin (a t), a \in R$

(4) $T_{\propto}(\tan (a t))=a t^{1-\propto} \sec ^{2}(a t), a \in R$

(5) $T_{\propto}(\cot (a t))=-a t^{1-\propto} \csc ^{2}(a t), a \in R$

(6) $T_{\propto}(\sec (a t))=a t^{1-\propto} \sec (a t) \tan (a t), a \in R$

(7) $T_{\propto}(\csc (a t))=-a t^{1-\propto} \csc (a t) \cot (a t), a \in R$

(8) $T_{\propto}\left(\frac{1}{\propto} t^{\propto}\right)=1$

Proof:

$1-T_{\propto}\left(e^{c x}\right)=\lim _{\varepsilon \rightarrow 0} \frac{e^{c\left(t+\varepsilon t^{1-\alpha}\right)}-e^{c t}}{\varepsilon}=e^{c t} \lim _{\varepsilon \rightarrow 0} \frac{e^{c \varepsilon t^{1-\alpha}}-1}{\varepsilon}$

$=e^{c t} \lim _{\varepsilon \rightarrow 0} \frac{t^{1-\alpha} e^{c \varepsilon t^{1-\alpha}}-t^{1-\alpha}}{\varepsilon t^{1-\alpha}}=t^{1-\propto} e^{c t} \lim _{\varepsilon \rightarrow 0} \frac{e^{c \varepsilon t^{1-\alpha}}-1}{\varepsilon t^{1-\alpha}}$

Let $\mathrm{h}=\varepsilon t^{1-\alpha}$

Then by using L 'Hopital' s rule, we get

$=t^{1-\propto} e^{c t} \lim _{h \rightarrow 0} \frac{e^{c h}-1}{h}=c t^{1-\propto} e^{c t} \lim _{h \rightarrow 0} \frac{e^{c h}}{1}=c t^{1-\propto} e^{c t}$

Corollary1: (Conformable fractional derivative of certain functions)

1) $T_{\propto}\left(\sin \frac{1}{\alpha} t^{\propto}\right)=\cos \frac{1}{\alpha} t^{\propto}$

2) $T_{\propto}\left(e^{\frac{1}{\alpha} t^{\alpha}}\right)=e^{\frac{1}{\alpha} t^{\alpha}}$

Note that the function could be $\alpha$-differentiable at a point but not differentiable. For example, let $f(t)=2 \sqrt{t}$. Then

$T_{\frac{1}{2}}(f)(0)=\lim _{t \rightarrow 0} T_{\frac{1}{2}}(f)(t)=1$, when $T_{\frac{1}{2}}(f)(t)=1$, for all $t>0$,

but $T_{1}(f)(0)$ does not exist

The most important case for the range of

$\mathrm{A} \in(0,1)$, when $\propto \in(n, \mathrm{n}+1)$ the definition would be as the following

Definition 5:let $\alpha \in(n, \mathrm{n}+1)$ and $f$ be an $\mathrm{n}$-differentiable at $\mathrm{t}$, where $\mathrm{t}>0$, then the conformable fractional derivative of $f$ of order $\alpha$ is defined as:

$T_{\alpha}(f)(t)=\lim _{\varepsilon \rightarrow 0} \frac{f^{([\alpha]-1)}(t+\varepsilon t([\alpha]-\alpha))-f^{([\alpha]-1)}(t)}{\varepsilon}$

Where $[\alpha]$ is the smallest integer greater than or equal to $\alpha$.

Theorem 4: (Rolle's Theorem for Conformable Fractional Differentiable Functions) 
Let $\alpha>0$ and $f:[a, b] \rightarrow R$ be a given function that satisfies

1- $f$ is continuous on $[\mathrm{a}, \mathrm{b}]$,

2- $f$ is $\alpha$-differentiable for some $\alpha \in(0,1)$,

3- $f(a)=f(b)$. then, there exists $C \in(\mathrm{a}, \mathrm{b})$, such that $f^{(\alpha)}(c)=0$.

Proof [2]: Since $f$ is continuous on [a,b], and $f(a)=f(b)$, there is $c \in(\mathrm{a}, \mathrm{b})$, which is a point of local extrema. With no loss of generality, assume $\mathrm{c}$ is a point of local minimum,so

$f^{\propto}(c)=\lim _{\varepsilon \rightarrow 0^{+}} \frac{f\left(c+\varepsilon c^{1-\alpha}\right)-f(c)}{\varepsilon}=\lim _{\varepsilon \rightarrow 0^{-}} \frac{f\left(c+\varepsilon c^{1-\alpha}\right)-f(c)}{\varepsilon}$

, but the first limit is non- negative, and the second limit is non-positive .Hence, $f^{\propto}(c)=0$.

\section{Applications}

Now in this section we will solve fractional differential equations according to conformable definitions:

Example 1:Let

$y^{1 / 2}+y=x^{3}+3 x^{5 / 2}, y(0)=0$

To find

$y_{h}$ of $y^{1 / 2}+y=0$

We use $y_{h}=e^{r \sqrt{x}}$

Now $y^{1 / 2}+y=0$

$e^{r \sqrt{x}}\left(\frac{r}{2}+1\right)=0$

$$
\frac{r}{2} e^{r \sqrt{x}}+e^{r \sqrt{x}}=0
$$

$\frac{r}{2}+1=0$ so $r=-2$

$y_{h}=e^{-2 \sqrt{x}}$

And simple the particular solution is $y_{p}=x^{3}$ and by plugging the initial condition

$y_{p}=x^{3}$ then $\mathrm{A}=0 \rightarrow y=y_{h}+y_{p}=e^{-2 \sqrt{x}}+x^{3}$

\section{Example 2:}

$y^{1 / 2}+y=x^{2}+2 x^{3 / 2}, \quad y(0)=0$

This is the equation that we mentioned in the introduction, approximating

$\Gamma(2.5)=1.33$ to 1

Let us find a solution $y_{h}$ of the homogeneous equation $y^{1 / 2}+y=0$, we look for a solution of the form

The auxiliary equation is

$$
y_{h}=e^{r \sqrt{x}} y^{\frac{1}{2}}+y=0 \rightarrow \frac{r}{2} e^{r \sqrt{x}}+e^{r \sqrt{x}}=0 \rightarrow \frac{r}{2}+1=0 \text { so } r=-2
$$

$y_{h}=e^{-2 \sqrt{x}}$

It is easy to verify that $y_{p}(x)=x^{2}$ is a particular solution of the nonhomogeneous equation. Now the general solution is

$y(x)=y_{h}(x)+y_{p}(x)=A e^{-2 \sqrt{x}}+x^{2}$

Where A is a constant. Finally the initial condition $y(0)=0$ implies that

$A=0 \operatorname{Hense} y(x)=x^{2}$

we should remark here that such an equation is a knwn one that people solve using the R-L derivative, with $\frac{2}{\Gamma(2.5)} x^{\frac{3}{2}}$ replacing $2 x^{\frac{3}{2}}$ and we get the same solution, but our method using our definition is much more easier.

Example 3: one can easily show that the auxiliary equation for $y^{\alpha}+y=0,0 \leq \alpha \leq 1, \propto r+1=0$ so that the solution is given by $y(x)=e^{-\frac{1}{\alpha} x^{\alpha}}$. more details including the method of undetermined coefficients will be discussed in a forthcoming paper.

in the following example we will show the benefit of the fractional derivative product rule which allows us to use the idea of the integrating factor.

\section{Example 4 :}

$y^{1 / 2}+\sqrt{x} y=x e^{-x}$

We solve this equation by multiplying it by $e^{x}$ then $e^{x} y^{1 / 2}+e^{x} \sqrt{x} y=x e^{x} e^{-x}$

$\boldsymbol{e}^{x} \boldsymbol{y}^{\mathbf{1} / 2}+\boldsymbol{e}^{\boldsymbol{x}} \sqrt{\boldsymbol{x}} \boldsymbol{y}=\mathbf{x} \rightarrow\left(e^{x} y\right)^{\frac{1}{2}}=x \rightarrow e^{x} y(x)=\frac{2}{3} x^{\frac{3}{2}}+C \rightarrow y(x)=\frac{2}{3} x^{\frac{3}{2}} e^{-x}+C e^{-x}$ 
$\mathrm{C}$ is constant, which can be a solution of the above equation.

\section{Conclusion}

The objective of the paper is to use "conformable fractional derivative" which is simpler and more efficient .The new definition reflects a natural extension of normal derivative to solve fractional differential equation.In this paper note that the derivative presented here does not have any delay effect but the other fractional ones have( there is a kernel in the definition inside the integral) .

Consider the very simple differential equation $y^{1 / 2}+y=0$.if one has to solve it using the Caputo or Riemann-Liouville definition, then he or she must use either the Laplace transform or the fractional power series technique. However, using conformable fractional derivative and the fact $T_{\propto}\left(e^{\frac{1}{\alpha} t^{\alpha}}\right)=e^{\frac{1}{\alpha} t^{\alpha}}$, one can easily see that $\mathrm{y}=\mathrm{c} e^{-2 \sqrt{t}}$ is the general solution.

According to one of the classical definitions. $D^{1 / 2}(\sin a x)=a^{1 / 2} \sin \left(a x+\frac{\pi}{4}\right)$. So what if a=-1 ? Now according to our definition $T_{\propto}\left(\sin \frac{1}{\alpha} t^{\propto}\right)=\cos \frac{1}{\alpha} t^{\propto}$. to evaluate $D^{\propto}\left(\sin \frac{1}{\alpha} t^{\propto}\right)$ using the classical definitions, it is not an easy job.

\section{Reference}

[1]. Boyce W. E., DiprimaR. C.,(2001),"Elementary Differential Equations and Boundary Value Problems." , (Seventh Edition), John Wiley \& Sons, Inc, new york, p. 259-300.

[2]. Abdeljawad, T.,(1015), "On Conformable Fractional Calculus." Journal of computational and Applied Mathematics, ELSEVIER, vol. 279, p. 57-66.

[3]. Ishteva, M. K., M.Sc. Thesis,(2005)" Properties and Applications of the Caputo Fractional Operator", University Karlsruhe, Bulgaria, pp. 16-30.

[4]. Shantanu D.,(2011), "Functional Fractional Calculus", Springer-Verlag,Berlin, Heidelberg, p. 10-50.

[5]. Khalil R., Al-Horani M., Yousef A., Sababheh M., (2014), "A New Definition of Fractional Derivative", Journal of Computational and Applied Mathematics, vol.264, pp.65-70.

[6]. Kisela T.,(2008), Diploma Thesis"Fractional Differential Equations and Their Applications", Brno University of Technology, pp. 13-50.

[7]. Podlubny I., (1998)," Fractional differential equations: an introduction to fractional derivatives, fractional differential equations, to methods of their solution and some of their applications", Academic press, vol. 198 ,p. 30-90.

[8]. Hilfer R.,(2002),"Applications of Fractional Calculus in Physics",: World Scientific publishing Co. Pte. Ltd., USA, p. 20-70.

[9]. Abo Hammad M., Khalil R.,(2014), "Abel's Formula and Wronskian for Conformable Fractiona Differential Equations", International Journal of Differential Equations and Applications, vol.13, no. 3, pp. 177-180.

[10]. Sebah P., Gourdon X., (2002),"Introduction to Gamma Function", American Journal of Scientific Research ,pp. 2-18. 